\title{
Characterization of 3D-printed lenses and diffraction gratings made by DLP additive manufacturing
}

\author{
Laura D. Vallejo-Melgarejo \\ Department of Mechanical Engineering, Universidad Nacional de Colombia, Bogotá, Colombia \\ Ronald G. Reifenberger \\ Department of Physics and Astronomy, Purdue University, West Lafayette, Indiana, USA \\ Brittany $A$. Newell \\ School of Engineering Technology, Purdue University, West Lafayette, Indiana, USA \\ Carlos A. Narváez-Tovar \\ Department of Mechanical Engineering, Universidad Nacional de Colombia, Bogotá, Colombia, and \\ Fosé M. Garcia-Bravo \\ School of Engineering Technology, Purdue University, West Lafayette, Indiana, USA
}

\begin{abstract}
Purpose - An Autodesk Ember three-dimensional (3D) printer was used to print optical components from Clear PR48 photocurable resin. The cured PR48 was characterized by the per cent of light transmitted and the index of refraction, which was measured with a prism spectrometer. Lenses and diffraction gratings were also printed and characterized. The focal length of the printed lenses agreed with predictions based on the thin lens equation. The periodicity and effective slit width of the printed gratings were determined from both optical micrographs and fits to the Fraunhofer diffraction equation. This study aims to demonstrate the advantages offered by a layer-by-layer DLP printing process for the manufacture of optical components for use in the visible region of the electromagnetic spectrum.

Design/methodology/approach - A 3D printer was used to print both lenses and diffraction gratings from Standard Clear PR48 photocurable resin. The manufacturing process of the lenses and the diffraction gratings differ mainly in the printing angle with respect to the printer $x$ - $y$-axes. The transmission diffraction gratings studied here were manufactured with nominal periodicities of 10,25 and $50 \mu \mathrm{m}$. The aim of this study was to optically determine the effective values for the distance between slits, $d$, and the effective width of the slits, $w$, and to compare these values with the printed layer thickness.

Findings - The normalized diffraction patterns measured in this experiment for the printed gratings with layer thickness of 10, 25 and $50 \mu \mathrm{m}$ are shown by the solid dots in Figures 8(a)-(c). Also shown as a red solid line are the fits to the experimental diffraction data. The effective values of $d$ and $w$ obtained from fitting the data are compared to the nominal layer thickness of the printed gratings. The effective distance between slits required to fit the diffraction patterns are well approximated by the printed layer thickness to within 14, 4 and 16 per cent for gratings with a nominal 10, 25 and $50 \mu \mathrm{m}$ layer thickness, respectively.

Research limitations/implications - Chromatic aberration is present in all polymer lenses, and the authors have not attempted to characterize it in this study. These materials could be used for achromatic lenses if paired with a crown-type material in an achromatic doublet configuration, because this would correct the chromatic aberration issues. It is worthwhile to compare the per cent transmission in cured PR48 resin (approximately 80 per cent) to the percent transmission found in common optical materials like BK7 (approximately 92 per cent) over the visible region. The authors attribute the lower transmission in PR48 to a combination of surface scattering and increased absorption. At the present time, the authors do not know what fraction of the lower transmission is related to the surface quality resulting from sample polishing.

Practical implications - There are inherent limitations to the 3D manufacturing process that affect the performance of lenses. Approximations to a curved surface in the design software, the printing resolution of the Autodesk Ember printer and the anisotropy due to printing in layers are believed to be the main issues. The performance of the lenses is also affected by internal imperfections in the printed material, in particular the presence of bubbles and the inclusion of debris like dust or fibers suspended in air. In addition, the absorption of wavelengths in the blue/ultraviolet produces an undesirable yellowing in any printed part.
\end{abstract}

The current issue and full text archive of this journal is available on Emerald Insight at: www.emeraldinsight.com/1355-2546.htm

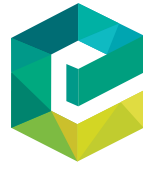

Rapid Prototyping Journal

25/10 (2019) 1684-1694

Emerald Publishing Limited [ISSN 1355-2546]

[DOI 10.1108/RPJ-03-2019-0074]
(C) Laura D. Vallejo-Melgarejo, Ronald G. Reifenberger, Brittany A. Newell, Carlos A. Narváez-Tovar and José M. Garcia-Bravo. Published by Emerald Publishing Limited. This article is published under the Creative Commons Attribution (CC BY 4.0) licence. Anyone may reproduce, distribute, translate and create derivative works of this article (for both commercial and noncommercial purposes), subject to full attribution to the original publication and authors. The full terms of this licence may be seen at http://creativecommons. org/licences/by/4.0/legalcode

Received 16 March 2019

Revised 14 June 2019

Accepted 29 July 2019 
Originality/value - One of the most interesting results from this study was the manufacture of diffraction gratings using 3D printing. An analysis of the diffraction pattern produced by these printed gratings yielded estimates for the slit periodicity and effective slit width. These gratings are unique because the effective slit width fills the entire volume of the printed part. This aspect makes it possible to integrate two or more optical devices in a single printed part. For example, a lens combined with a diffraction grating now becomes possible.

Keywords Resin PR48, 3D-printed lenses, DLP additive technology, Diffraction gratings, 3D-printed diffraction gratings

Paper type Research paper

\section{Introduction}

Additive manufacturing has seen significant advancements in materials and processes over the past decade. However, it is minimally used for production of optical parts. Fused deposition modeling (FDM also known as fused filament fabrication) is a relatively inexpensive, very common and simple method for prototyping parts. Clear materials like polyethylene terephthalate (PETG) and polycarbonate (PC) may be used to print lenses or other optical devices. It is known that better optical results can be achieved using vat through all document at polymerization, an additive method where a photo-polymer resin is cured using a light source (typically UV light). The most popular techniques at polymerization threedimensional (3D) printing are stereolithography (SLA) and digital light processing (DLP) (Burns, 1993). Of these two methods, DLP additive manufacturing is preferred because it has a nominal layer resolution of approximately $50 \mu \mathrm{m}$, with some printers being able to print parts with a resolution as low as $10 \mu \mathrm{m}$ (Burns, 1993). FDM, on the other hand, has a resolution higher than $100 \mu \mathrm{m}$, and the printed parts are more susceptible to warping, which makes this method less desirable for optical components.

Literature reveals many approaches to novel fabrication methods for production of optical components without resorting to traditional grinding and polishing methods. Two generic methods have generally been followed. One methodology emphasizes a self-assembly theme. For example, short focal length lenses (f approximately $6 \mathrm{~mm}$ ) have been fabricated by exploiting surface tension and the fluid viscosity of ink-jet deposited droplets to form flexible lenses that attach to smartphones to produce $120 \times$ magnified images (Sung et al., 2015). A second approach utilizes $3 \mathrm{D}$ printing technology to directly fabricate optical components. An example of this approach comes from researchers at Rice University (Gawedzinski et al., 2017) who have carefully compared the optical characteristics of traditionally machined glass lenses to 3D-printed lenses using the Printoptical Luxexcel technology which relies on the jetting of droplets of a UV-curable resin. They determined that the root mean square wavefront error of the printed lenses was approximately 19 times larger than an equivalent glass lens with a $12.7 \mathrm{~mm} 100$ per cent clear aperture. However, when the two lenses were compared at 63 per cent of its clear aperture, the 3D-printed prototypes gave a comparable wavefront error. They also reported moderately larger refractive indices for the 3D-printed lenses when compared to typical polymethyl methacrylate (PMMA) lenses. Other researchers (Chen et al., 2018) demonstrated a method for fabricating a customized aspherical lens $5 \mathrm{~mm}$ in thickness and $3 \mathrm{~mm}$ in diameter. In their study, the quality of the $3 \mathrm{D}$-printed lenses was dramatically improved by reducing the voxel size. For their experiment, the researchers used a femto-second 3D printing process utilizing a highly parallel projection micro-SLA process, producing final parts with resolutions as low as $100 \mathrm{~nm}$ length scale. This method enables cameras in mobile phones to be used as low-cost microscopes capable of sub-millimeter magnification.

It is not surprising that the fabrication of the 3D-printed terahertz (THz) optical components has been explored. In the $\mathrm{THz}$ region of the electromagnetic spectrum, the relevant wavelengths are measured in fractions of a millimeter rather than 100 s of nanometers, making the realization of geometric tolerances more favorable to $3 \mathrm{D}$ printing. As an example, Squires and his group (Squires et al., 2015) have reported the printing of both regular and blazed conventional diffraction gratings and aspherical lenses for use at $\mathrm{THz}$ frequencies using a fused filament technique. Their work showed the measured optical performance matched theoretical expectations. Similarly, Furlan et al. (2016) characterized the 3D-printed $\mathrm{THz}$ diffractive lenses. This work utilized an online 3D printing service that fabricated parts from a polyamide granular powder using a selective laser sintering technique. According to these authors, additive manufacturing is highly suitable for manufacturing these types of lenses because it eases the construction of nonconventional fractal, Fibonacci and $\mathrm{THz}$ binary diffractive lenses and produces satisfactory results when compared to commercially available products.

The prior work on 3D-printed optical components (Gawedzinski, 2017; Chen, 2018; Squires, 2015; Furlan, 2016) does not explore at polymerization methods which are widely available and accessible. This topic is worth investigating because a distinctive characteristic at polymerization is the smooth surface finish and fine feature details it can produce. For this reason, the present study will take advantage of the high spatial resolution achieved by DLP technology to fabricate optical lenses and gratings using at polymerization. The aim of this work is to assess the quality and performance of the 3D-printed at-polymerized optical components and to learn what if any new optical functionality they might produce.

\section{Manufacture and processing considerations}

It is known that the quality and material properties of final parts produced using DLP as well as other additive manufacturing processes depend on multiple manufacturing parameters (Li et al., 2017). For example, Monzón et al. (2017) studied the influence of build direction and post-curing processes on 
mechanical properties and the anisotropy of DLP parts using three different photopolymer resins (Castable Blend, Visijet FTX Green and Industrial Blend). They demonstrated that the pixelation in DLP parts is the fundamental cause of anisotropy between vertical and horizontal build directions. Riahi (2016) studied the effect of the printing direction and layer thickness on the surface quality of DLP corner cube array retro-reflective structures. He also proposed a thermal reflow post-processing technique as a surface finishing process and claimed that this technique could also be used to manufacture other optical elements such as micro-lens arrays.

Other parameters relate directly to the printing material. Gong et al. (2015) investigated the effect of resin optical properties on the minimum void size that can be fabricated using DLP. Their results showed that an optimized curing depth of a resin part may be achieved by increasing resin absorbance and $x-y$ plane resolution of the projected image. In addition, Lee et al. (2001) demonstrated the existence of a critical photoinitiator concentration that optimizes the curing depth of a resin.

In the study presented here, an Autodesk Ember 3D printer was used to print both, lenses and diffraction gratings, from Standard Clear PR48 photocurable resin. The manufacturing process of the lenses and the diffraction gratings differ mainly in the printing angle with respect to the printer $x-y$ axes (the printbed). The value of this angle for the lenses and the diffraction gratings is based on the results obtained in a previous study (Melgarejo et al., 2018) where it was shown that light diffraction occurs in parts with printing angles greater than $60^{\circ}$ with respect to the printbed. Diffraction is more noticeable as the printing angle increases. For this reason, lenses are printed at $0^{\circ}$ and the diffraction gratings are printed at $90^{\circ}$ with respect to the printbed. Lenses were printed with a layer thickness of $25 \mu \mathrm{m}$, while the diffraction gratings were varied to have layer thickness of 10, 25 and $50 \mu \mathrm{m}$. A schematic of the optical components investigated in this study showing the orientation of the printed layers is given in Figure 1.

The general fabrication process follows the steps in a previous article (Melgarejo et al., 2018) and is described as follows:

- modeling of optical components in Autodesk Inventor 2017;

- generation of layers of the model (slicing) using PrintStudio;

Figure 1 Schematic diagram of the optical components printed

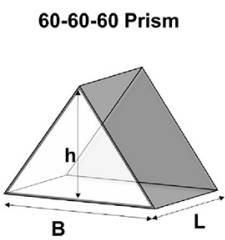

(a)

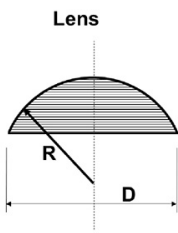

(b)

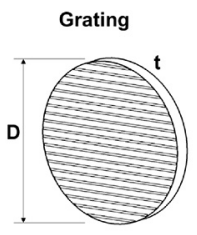

(c)
Notes: The orientation of the printed layers for the grating and lens are indicated. Typical dimensions: in (a) $\mathrm{B}=30 \mathrm{~mm}, \mathrm{~h}=26 \mathrm{~mm}, \mathrm{~L}=30 \mathrm{~mm}$; in (b) $\mathrm{R}=25 \mathrm{~mm}, \mathrm{D}=30 \mathrm{~mm}$; in (c) $\mathrm{t}=2.5 \mathrm{~mm}$, $\mathrm{D}=30 \mathrm{~mm}$
- printing the optical components using the Autodesk Ember 3D printer and PR48 resin;

- smoothing the components using sandpaper with grits from 800 to 3,000; and

- polishing the components with polishing cloth, using a three-step commercial polishing agent and scratch removal kit for plastic acrylic and fiberglass.

The polishing process typically produced an average roughness of $33 \mathrm{~nm}$ over a 100 square micron area as determined from the atomic force microscope images.

The Ember 3D printer uses an LED light with a wavelength of $405 \mathrm{~nm}$ and $5 \mathrm{~W}$ of optical power to cure the resin. Its resolution in the $\mathrm{x}-\mathrm{y}$ plane is $50 \mu \mathrm{m}$ with a vertical step along the $\mathrm{z}$-axis between 10 and $100 \mu \mathrm{m}$ (Aniwaa, 2019). It is known that a DLP printer allows variation of more than 15 parameters to control the printing process. The default parameters used in this study are listed in Table I and remained constant for all printed optical components. The most important parameter in our study is the layer thickness because it controls the periodicity of our printed optical components. In the present work, this one parameter was varied to study its effects on optical quality. The corresponding exposure time for each layer thickness follows that recommended by the printer manufacturer. The exposure times according to the layer thickness are given in Table II.

In this work, AutoDesk PR48 Clear resin containing two oligomers, a photoinitator, a reactive dilutent and a UV blocker was used (Autodesk, 2018). The oligomers in this resin are Allnex Ebecryl 8210 (39.776 Wt.\%) and Sartomer SR 494 (39.776 Wt.\%). Ebecryl 8210 is an aliphatic urethane acrylate with a low viscosity and high reactivity to ultraviolet (UV) radiation (Allnex, 2013). Urethane acrylates are high molecular weight materials generally used for cure in place applications. These materials exhibit flexibility, toughness, chemical resistance and yellowing. Sartomer SR 494 is an alkoxylated pentaerythritol tetraacrylate. SR 494 is a multifunctional acrylic

Table I Default parameters of the printer

\begin{tabular}{ll}
\hline Setting & Value \\
\hline Wait before exposure & $15 \mathrm{~s}$ \\
Separation slide velocity & $8.0 \mathrm{rpm}$ \\
Z-axis overlift & $0.75 \mathrm{~mm}$ \\
Separation Z-axis velocity & $1.5 \mathrm{~mm} / \mathrm{s}$ \\
Approach slide velocity & $12.0 \mathrm{rpm}$ \\
Approach Z-axis velocity & $1.5 \mathrm{~mm} / \mathrm{s}$ \\
Angle of rotation & $60^{\circ}$ \\
Overpress & $0.0 \mathrm{~mm}$ \\
Overpress velocity & $1.5 \mathrm{~mm} / 2$ \\
Approach/separation slide max jerk & $4,629^{\circ} / \mathrm{s}^{3 *}\left(\times 10^{3}\right)$ \\
Approach/separation Z-axis max jerk & $0.046 \mathrm{~cm} / \mathrm{s}^{3}$ \\
\hline
\end{tabular}

Table II Exposure time for varying layer thickness

\begin{tabular}{lc}
\hline Layer thickness $(\mu \mathrm{m})$ & Exposure time $(\mathrm{s})$ \\
\hline 10 & 1.8 \\
25 & 2.0 \\
50 & 2.2 \\
\hline
\end{tabular}


monomer that can be polymerized by free radicals (Arkema, 2014).

PR48 contains one reactive monomer, Rahn Genomer 1122 (19.88 Wt.\%). This material serves to reduce the viscosity and crosslink in the final cured resin. The photoinitiator, Esstech $\mathrm{TPO}+(0.4 \mathrm{Wt} . \%)$ serves as a catalyst to initiate the reaction at $405 \mathrm{~nm}$ light. Free radicals, formed from breakdown of the photoinitiator, initiate the cross-linking process of the monomers and oligomers. The UV blocker, Mayzo OB+ $(0.16 \mathrm{Wt} . \%)$ controls UV penetration which confines the cured layer thickness (Skliutas et al., 2018).

Within these bonded structures, several parameters can affect the formation of microvoids inherent to the 3D-printed structures and particularly to the 3D-printed lens. These voids or channels which directly affect print resolution are affected by the optical dose received and the exposure between layers (Gong et al., 2015). In areas where overexposure occurs, photopolymerization lasts for a longer time resulting in wider overall features (Skliutas et al., 2018). In the current study, optical dosing is set to standard conditions for PR48 in the Ember printer (see parameters in Table II). It is known that cavity dimensions are controlled by the optical dose and the original inherent chemistry, particularly the UV photo blocker controlling light penetration between layers (Riahi, 2016). For this reason, the authors expect that these factors to be relevant at the interface between two layers.

\section{Optical characterization}

To characterize optical components, such as lenses and diffraction gratings, it is necessary to know the interaction of the light with the printing material of these elements. In this section, the refractive index and the per cent transmission were experimentally determined at different wavelengths spanning the visible region of the electromagnetic spectrum.

\subsection{Refractive index}

\subsubsection{Theory}

The refractive index is a complex and dimensionless number that characterizes a medium by relating the speed of propagation of light in vacuum to the speed of light through the material. Its value varies depending on the wavelength of the incident light (Pedrotti et al., 2017).

A method to determine the real part of the refractive index of a material requires a prism made from the material to be tested. When a light beam passes through the prism, its direction changes with an angle of deviation $\delta(\lambda)$, which varies for each wavelength that composes the incident light ray, as shown in Figure 2. The angle of deviation depends on the apical angle $\alpha$ of the prism, its refractive index and the initial angle of incidence. The angle of deviation has a minimum value $\delta_{\min }(\lambda)$ when the light at wavelength $\lambda$ passes symmetrically through the prism (Freeman and Hull, 2003). When this condition is met, the index of refraction can be accurately calculated from equation (1):

$$
n(\lambda)=\frac{\sin \left[\frac{1}{2}\left(\alpha+\delta_{\min }(\lambda)\right)\right]}{\sin \left(\frac{\alpha}{2}\right)} .
$$

The minimum deviation angle must be known to use equation (1), and it must be determined experimentally for
Figure 2 A schematic showing different collimated rays through a prism with an apex angle $\alpha$

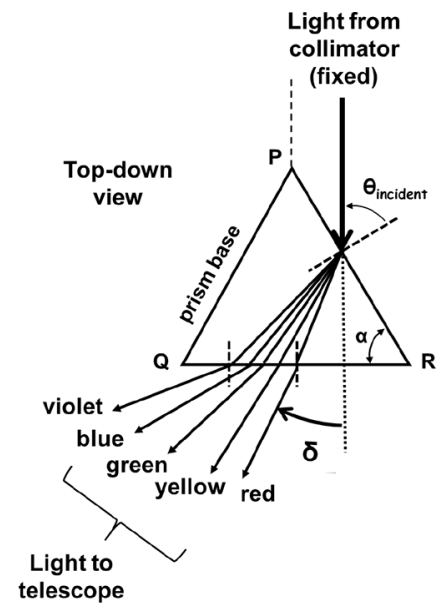

Notes: As the prism is rotated to change $\theta_{\text {incident }}$, the angle of deflection $\delta$ varies. When $\delta$ reaches a minimum value, the path of the ray through the prism becomes parallel to the prism base. This condition permits an accurate measurement for $\mathrm{n}$, the index of refraction of the prism at different wavelengths of light

each wavelength. If measurements of $\delta_{\min }(\lambda)$ are made at a few wavelengths, it is convenient to use an expression that allows an interpolation of $n$ for any wavelength. The empirical Cauchy formula, given in equation (2), is often used:

$$
n(\lambda)=C_{1}+\frac{C_{2}}{\lambda^{2}}+\ldots
$$

The constants $C_{1}$ and $C_{2}$ are determined from a least squares fit of the data.

The material from which the prism is manufactured can be classified according to its dispersive power using a glass map. To use this map, it is necessary to know in advance the refractive index at well-prescribed wavelengths, which can then be used to define the Abbe number $V$ of the material. The Abbe number is determined by equation (3) (Hecht, 2017):

$$
V \equiv \frac{n_{d}-1}{n_{f}-n_{c}}=\frac{n(587.6)-1}{n(486.1)-n(656.3)}
$$

\subsubsection{Methodology}

To determine the refractive index of the material, it is necessary to measure the angle of minimum deviation $\delta_{\text {min }}(\lambda)$. In this study, we used a prism spectrometer, a helium discharge tube and a prism manufactured using the photocurable resin. The spectrometer had four main parts: a table for supporting the prism, a collimator equipped with an adjustable slit, a viewing telescope and a Vernier scale with a least count (main scale division/number of divisions) of $1 \mathrm{~min}$ of arc. The triangular 
prism was manufactured (not printed) with PR48 resin, cured with a UV lamp with a wavelength of $405 \mathrm{~nm}$ and subsequently polished and buffed. After processing, all apical angles $\alpha$ were measured at $60^{\circ}$ as determined by a protractor. The experimental setup used for testing is schematically shown in Figure 3.

An accurate measurement of the angle of minimum deviation requires a careful adjustment of the prism spectrometer. The collimator slit was illuminated by the He discharge tube and the telescope was focused and aligned with the collimator so that an image of the slit could be seen through the eyepiece of the telescope (Freeman and Hull, 2003). The reading of the Vernier in this position of the telescope was set to zero and was taken as a reference point.

The prism was placed on the table with one refracting face making an angle of approximately $45^{\circ}$ with the beam from the collimator (Freeman and Hull, 2003). Then, the telescope was rotated until a sequence of lines (corresponding to the characteristic wavelengths of the $\mathrm{He}$ spectrum in the visible range) was observed through the telescope. The prism was then slowly rotated until the minimum angle of deviation was observed. The angle of the telescope was then successively adjusted to align with each line, and each position was measured with the Vernier. Six independent measurements for each of the observable wavelengths were made.

The color of each line observed through the telescope corresponded to a characteristic and well-defined wavelength in the $\mathrm{He}$ visible spectrum (Table III). This allowed a correlation between the measured angles of minimum deviation with a known wavelength.

Subsequently, equation (1) was used to find the refractive index from the measured angles of minimum deviation. These data were then fit to the empirical Cauchy equation (equation (2)) to obtain an equation that related any wavelength of the material with its correspondent refractive index. Finally, equation (3) was used to find the Abbe number of the material.

Figure 3 Experimental setup for measuring the refractive index

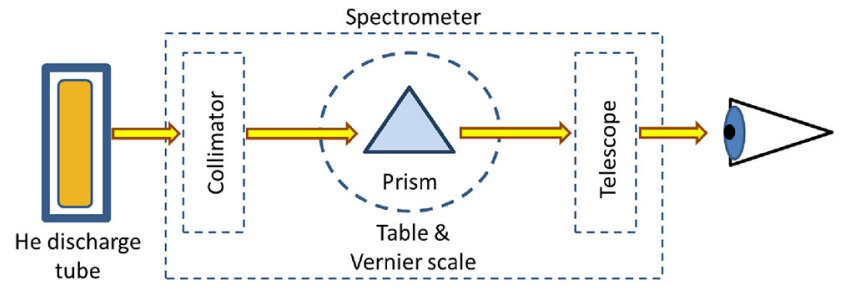

Table III Characteristic wavelengths of the He in the visible spectrum

\begin{tabular}{lc}
\hline Color & Wavelength $\lambda(\mathrm{nm})$ \\
\hline Deep blue & 447.1 \\
Blue & 471.3 \\
Blue-green & 492.2 \\
Green & 501.6 \\
Yellow & 587.6 \\
Red & 667.8 \\
Red & 706.5 \\
\hline
\end{tabular}

\subsubsection{Results}

When examining the light refracted by the prism made with PR48 resin, only five main wavelengths were recognized, and only four can be easily measured using the spectrometer. These wavelengths are shown in Table IV.

Equation (1) was used to determine the refractive index after measuring the minimum deviation angle $\delta_{\min }(\lambda)$ for the four observed wavelengths. The obtained values were presented in Table IV.

The coefficients in the Cauchy formula were determined by making a least squares fit to the data presented in the plot of the refractive index $n(\lambda)$ versus the reciprocal of the wavelength squared $\lambda^{-2}$, as shown in Figure 4.

The coefficients $C_{1}$ and $C_{2}$ corresponded to the intercept with the $y$-axis and the slope of the straight line, respectively, and the calculated values are:

$$
\begin{aligned}
& C_{1}=1.4919 \pm\left(8.3 \times 10^{-5}\right) \\
& C_{2}=5360.6 \pm(22.5) \mathrm{nm}^{2}
\end{aligned}
$$

These two coefficients made it possible to determine the refractive index for any wavelength in the visible spectrum. Finally, the Abbe number for PR48 resin was calculated from equation (3), which was found to be: $V=49.5$.

\subsection{Transmission}

\subsubsection{Theory}

Two factors that limit the transparency of a material are reflectivity and absorbance. When manufacturing optical components, a measurement of these values is useful. The light that passes through a transparent material is attenuated due to both extrinsic and intrinsic losses. Extrinsic losses are related to reflection and scattering from the surface. Intrinsic losses are due to absorption and scattering from molecules comprising

Table IV Refractive index for the PR48 prism at four known wavelengths

\begin{tabular}{lccc}
\hline Color & $\begin{array}{c}\text { Wavelength } \\
\lambda(\mathrm{nm})\end{array}$ & $\begin{array}{c}\text { Index of } \\
\text { refraction } n(\lambda)\end{array}$ & $\begin{array}{c}\text { Uncertainty } \\
( \pm)\end{array}$ \\
\hline Blue & 447.1 & 1.5187 & 0.0002 \\
Green & 501.6 & 1.5132 & 0.0002 \\
Yellow & 587.6 & 1.5074 & 0.0002 \\
Red & 667.8 & 1.5040 & 0.0003 \\
\hline
\end{tabular}

Figure $4 \mathrm{~A}$ least squares fit of the refractive index vs $\lambda^{-2}$ according to the Cauchy expansion (equation (2))

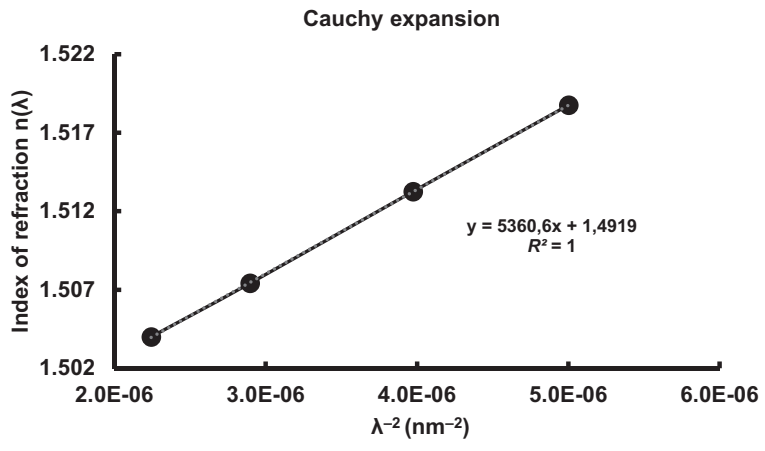


the core material and by residual (and unwanted) inclusions produced during sample fabrication.

For a transparent material, energy conservation considerations can be used to write:

$$
I_{0}=R+A+T
$$

where $I_{O}$ is the incident irradiance, and R, A and T measure the amount of light reflected, absorbed and transmitted, respectively. Each of these quantities depends on the wavelength $\lambda$. For the case of normal incidence, the value of the reflectance due to a dielectric discontinuity is given by Pedrotti et al. (2017):

$$
R=\left(\frac{n_{1}-n_{2}}{n_{1}+n_{2}}\right)^{2}
$$

where $n_{1}$ and $n_{2}$ are, respectively, the refractive index of the surrounding material (air) and the optical material of interest. Typically, for a single well-polished air/sample interface, $R / I_{0}$ is approximately 0.04 . The value of $R$ obtained from equation (5) can increase above this ideal value due to a variety of effects like surface roughness.

\subsubsection{Methodology}

To measure $T(\lambda)$ an OceanView Red Tide USB650 spectrometer was used, a Fiber-Lite (Model 190) fiber optic illuminator, a neutral density filter (NDF) with optical density of 1.0 and a slab made from cured PR48 resin with a thickness of $30.15 \pm 0.02 \mathrm{~mm}$ were needed. The PR48 slab was cured with a UV lamp with a wavelength of $405 \mathrm{~nm}$ and subsequently polished and buffed. The experimental arrangement is shown in Figure 5. For the measurement of $I_{0}(\lambda)$ and $T(\lambda)$, an integration time of $13 \mathrm{~ms}$ was used. Nine individual scans were averaged to obtain an experimental set of data.

\subsubsection{Results}

Figure 6 plots a typical percent transmission curve for the polymer sample studied. The data indicate a relatively featureless curve with approximately 80 per cent transmission between 475 and 1,000 $\mathrm{nm}$. The per cent transmission exhibits a sharp drop below $450 \mathrm{~nm}$, as the irradiance peak of the 3D printer is $405 \mathrm{~nm}$ (Aniwaa, 2019) and the resin must absorb the light at a similar wavelength for the curing process to take place. This will cause strong attenuation in the blue/violet region of the electromagnetic spectrum. Repetitive studies further showed the value of $T(\lambda)$ between 475 and $1,000 \mathrm{~nm}$ depended on the region of the sample illuminated, indicating that inhomogeneous surface polishing was likely an issue.

Figure 5 Experimental setup for measuring the attenuation coefficient

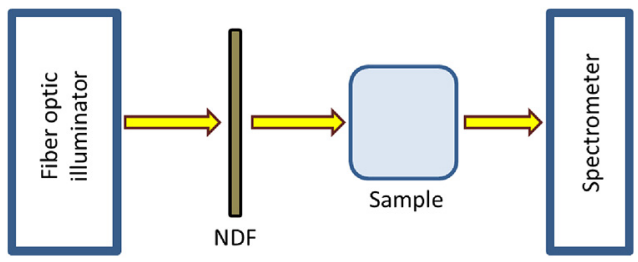

Figure 6 The per cent transmission in polymer PR48 at nominal thickness $30 \mathrm{~mm}$ for wavelengths between 400 and 1,000 nm

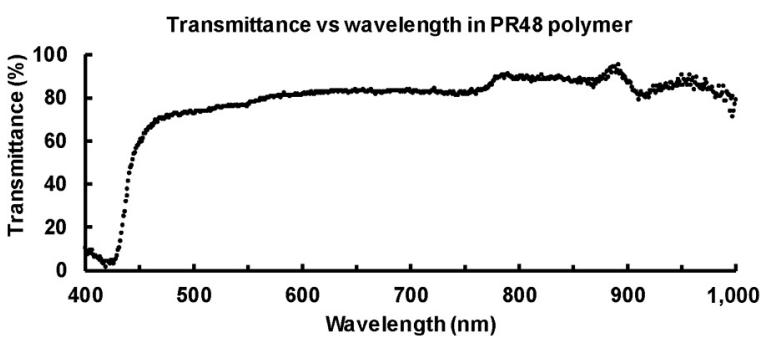

\section{Optical components}

Optical devices such as lenses and diffraction gratings manipulate electromagnetic waves and are widely used in the construction of optical systems (Pedrotti et al., 2017). This section focuses on determining the feasibility of manufacturing lenses and diffraction gratings, using DLP SLA, through the measurement of optical characteristics and the comparison of these results with theoretical models.

\subsection{Printing lenses}

4.1.1 Theory

In the case of thin lenses (lenses with a negligible thickness compared to the radii of curvature of the lens), the focal length can be approximated from the lensmaker's equation (Pedrotti et al., 2017):

$$
\frac{1}{f}=\frac{n_{2}-n_{1}}{n_{1}}\left(\frac{1}{R_{1}}-\frac{1}{R_{2}}\right)
$$

where $n_{1}$ and $n_{2}$ correspond to the refractive indices of the surrounding medium and the lens material, respectively, and $R_{1}$ and $R_{2}$ correspond to the radii of curvature of the two surfaces of the lens. In most cases, the environment is air, so $n_{1}=1$ (Pedrotti et al., 2017). In addition, when one of the faces of the lens is flat, its radius of curvature is taken as infinite, which makes $\frac{1}{R_{2}}=0$. Consequently, equation (6) can be simplified to:

$$
\frac{1}{f}=(n-1)\left(\frac{1}{R}\right)
$$

In this study, Bessel's method was used to measure the focal length of the printed lenses. This method is based on the principle of reversibility of light, which states that any actual ray of light in an optical system, if reversed in direction, will retrace the same path backward (Pedrotti et al., 2017). Therefore, the distance of the object to the lens when the image is in focus can be interchanged by the distance between the image and the lens, and a focused image will also be obtained.

In practice, the Bessel method requires the displacement of a lens along the optical axis between a fixed illuminated object and a fixed image screen. If the object and image screen are separated by a distance greater than four times the focal length of the lens, two positions of the lens will be found in which the image is in focus on the screen, magnified in one case and reduced in the other (Pedrotti et al., 2017). The focal length of the lens is then given by: 


$$
f=\frac{L^{2}-a^{2}}{4 L}
$$

where $L$ is the distance from the object to the screen, and $a$ corresponds to the distance between the two lens positions in which the image is in focus on the screen.

\subsubsection{Methodology}

A plano-convex lens with radii of curvature of $25 \mathrm{~mm}$ and thickness of $4 \mathrm{~mm}$ along the optical axis was manufactured using the PR48 resin. To minimize surface defects in the printed material, the lens was subjected to the polishing process described in Section 2 above. Equation (7), using a refractive index of $n=1.513$ (at $501.6 \mathrm{~nm}$ ), was used to calculate an expected focal length of the lens to be $48.7 \mathrm{~mm}$.

To measure the focal length of the lens, the object and the screen were placed on an optical rail and the lens was placed in between, as shown in Figure 7. The distance between the screen and object was kept constant, while the position of the lens was varied until a clear image of the object was produced on the screen. The position of the lens was changed until a second sharp image was observed.

Subsequently, the distance between the object and the screen $(L)$ and the distance between the two lens positions where a clear image on the screen was obtained $(a)$ were measured. Five repetitions were made to obtain an average value for $f$. Finally, the experimental focal length calculated from equation (8) was compared to the theoretical value of the focal distance.

\subsubsection{Results}

The theoretical focal length of the plano-convex lens, calculated from equation (7), was found to be $48.7 \mathrm{~mm}$. The experimental focal length, calculated from the measurements and the equation (8), was $48.4 \pm 0.4 \mathrm{~mm}$. From these results, it was determined that the focal length of the lens differs by 0.6 per cent from its theoretical value.

\subsection{Printing diffraction gratings}

\subsubsection{Theory}

Diffraction gratings are optical elements with multiple periodic slits, which disperse light through angles that depend on the wavelength of the incident light (Pedrotti et al., 2017). In the Fraunhofer limit, when $N$ slits are uniformly illuminated by incident light of irradiance $I_{0}$, the intensity pattern observed on a distant viewing screen is given by:

$$
I=I_{0}\left(\frac{\sin \beta}{\beta}\right)^{2}\left(\frac{\sin (N \alpha)}{\sin \alpha}\right)^{2}
$$

The arguments of the sine terms in equation (9) are given by:

$$
\beta=\frac{\pi w \sin \theta}{\lambda} \quad \alpha=\frac{\pi d \sin \theta}{\lambda}
$$

where $w$ equals the width of an individual slit, $d$ equals the distance between slits and $\lambda$ is the wavelength of the incident coherent light through the periodic slits. The angle between the optical axis and a specified position on a distant viewing screen is given by the angle $\theta$. For a fixed $\lambda$, the values of $w, d$ and $N$ control separate well-defined characteristics of the observed diffraction pattern on the viewing screen.

\subsubsection{Methodology}

Intrinsic to the $3 \mathrm{D}$ printing process, the thin interface between adjacent layers can possess a different optical transmission from the remainder of the layer, in effect creating a periodic array of slits. The transmission diffraction gratings studied here were manufactured following the process described in Section 2 above. Gratings with nominal periodicities of 10, 25 and $50 \mu \mathrm{m}$ were fabricated. The aim of this study was to optically determine effective values for the distance between slits $d$ and the effective width of the slits $w$ and to compare these values with the printed layer thickness.

To measure the diffraction pattern of light transmitted through the grating, an experiment was performed using a standard two-lens Fraunhofer arrangement. The laser beam from a red HeNe laser $(\lambda=632.8 \mathrm{~nm}$ ) was conditioned by a spatial filter and was rendered parallel by a near-field lens (Lens 1). The collimated light beam passed through a printed diffraction grating, and the diffracted beam was focused on a viewing screen by a far-field lens (Lens 2). A Pasco highsensitivity light sensor CI-6604, equipped with a $100 \mu \mathrm{m}$ wide slit, was scanned across the diffraction pattern at a speed of approximately $135 \mu \mathrm{m} / \mathrm{s}$, while the intensity profile was digitized by a computer. A schematic of the experiment is provided in Figure 8.

The diffraction data generated by each printed grating were fit using equation (9) by adjusting the values of $N, d$ and $w$. The parameter $w$ controls the width of the overall diffraction envelope (the central maximum), while the parameter $d$ controls the distance between interference peaks. Lastly, $N$ controls the width (sharpness) of each interference peak. These parameters were adjusted until a reasonable fit to the measured diffraction pattern was obtained.

Figure 7 Experimental setup for measuring the focal length

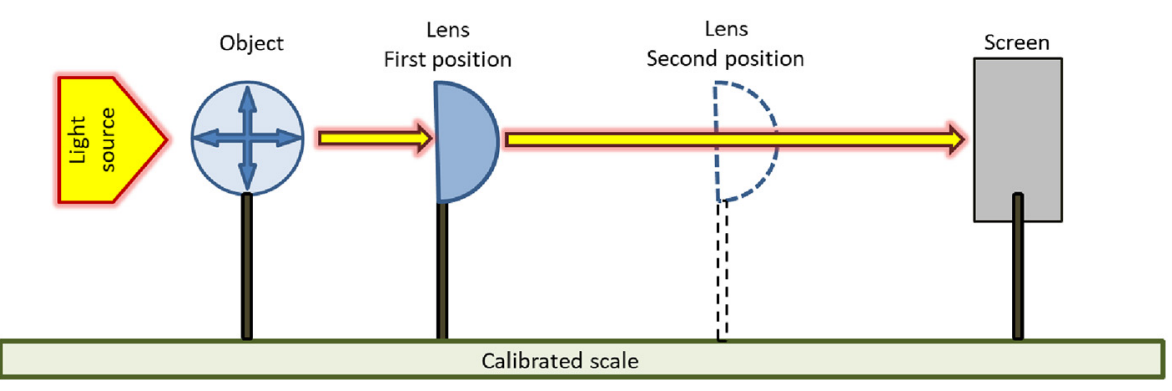


Figure 8 Experimental setup for measuring the diffraction pattern produced by a grating

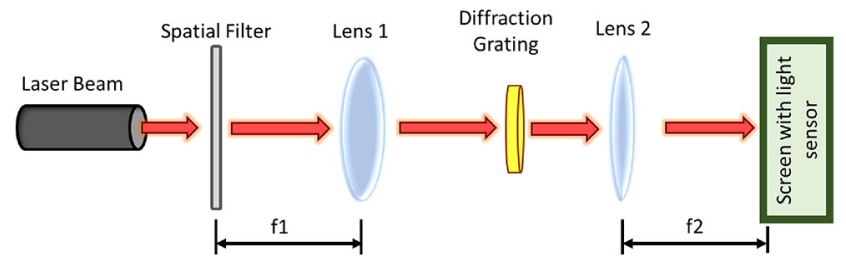

In a second experiment, the distance between slits $d$ and the width of the slits $w$ of the diffraction gratings were estimated optically using micrographs taken with the Eclipse LV100N POL microscope with a magnification of $500 \times$. A statistical approach based on a completely randomized design of fixed effects was used to determine the number of independent measurements required to achieve a specified confidence level. Input parameters to this statistical analysis required an a priori specification of a standard deviation that was considered acceptable. This value was set to equal to $0.8 \mu \mathrm{m}$. Three separate samples were specified (three printed gratings, each with a different periodicity). The true difference of the mean effect $(\alpha)$ was specified as 0.05 and a confidence level of 0.8 or greater was selected. Using these conditions, it was concluded that approximately 40 random measurements were required for each printed grating to obtain reliable estimates for $d$ and $w$.

\subsubsection{Results}

The normalized diffraction patterns measured in this experiment for the printed gratings with layer thickness of 10 , 25 and $50 \mu \mathrm{m}$ are shown by the solid dots in Figures 9 (a)-(c). Also shown as a red solid line are the fits to the experimental diffraction data. The effective values of $d$ and $w$ obtained from fitting the data are compared to the nominal layer thickness of the printed gratings as shown in Table V.

Table $\mathrm{V}$ shows that the effective distance between slits required to fit the diffraction patterns are well approximated by the printed layer thickness to within 14, 4 and 16 per cent for gratings with a nominal 10,25 and $50 \mu \mathrm{m}$ layer thickness, respectively.

Representative micrographs of the printed gratings taken with the optical microscope are shown in Figure 10(a)-(c). It should be noted that while performing this study, the optical filters in the microscope were adjusted to optimize the perceived optical contrast. This can produce an inverted pattern as clearly observed in Figure 10(c) where the narrow region characterizing the interface between printed layers appears dark rather than bright as might be expected for a transparent region of width $w$.

The optical focus in each micrograph was adjusted differently. In Figure 10(a), the focus was set below the surface of the printed grating to highlight the density and typical dimensions of inclusions below the surface. By contrast, in Figure 10(c), the microscope focus was set at the surface of the printed grating to high-lite surface scratches produced by polishing.

The average values for $d$ and $w$, estimated from measurements of these micrographs, are summarized in Table VI as $\langle d\rangle$ and $<w>$. The percent difference in $d$ between these values are $0,1.6$ and 0.4 per cent for the printed gratings with a nominal layer thickness of 10,25 and $50 \mu \mathrm{m}$, respectively.

Table VII shows the percentage difference between the values of $d$ and $w$ obtained from the diffraction data and the values measured from the optical micrographs. In the case of the distance between slits $d$, the percentage difference does not exceed 18 per cent of the value for the layer thickness of the printed grating. On the other hand, the slit width $w$ shows a considerable discrepancy for the 25 and $50 \mu \mathrm{m}$ gratings, while for $10 \mu \mathrm{m}$, this percentage does not exceed 10 per cent.

\section{Discussion}

In what follows, the results obtained above are evaluated within a broader context to learn what advantages or disadvantages the PR48 resin might offer when printing optical components.

\subsection{Optical characterization}

A slab of the cured PR48 resin was found to have an Abbe number of 49.5 and a refractive index of 1.507 (at a wavelength of $587.6 \mathrm{~nm}$ ). When compared to a glass map, the equivalent type of glass can be identified. It was concluded that the material belongs to the category of extra light flint (ELF). This indicates that the dispersed light (the mean deviation among the various wavelengths traversing the material) is greater in comparison with materials from the crown type. By only considering the dispersion power of the material, it can be concluded that the cured PR48 resin material is suitable for dispersive optical elements such as prisms, where high light dispersion is sought.

Some polymers in the "flint glass-equivalent" category are already used for the manufacture of lenses. Examples include: PC with a mean refractive index $=1.58$ and $\mathrm{V}=29.5$, and polystyrene with a mean refractive index $=1.59$ and $\mathrm{V}=30.5$ (Freeman and Hull, 2003). Chromatic aberration is present in all polymer lenses, and we have not attempted to characterize it in this study. These materials could be used for achromatic lenses if paired with a crown-type material in an achromatic doublet configuration, because this would correct the chromatic aberration issues.

It is worthwhile to compare the per cent transmission in cured PR48 resin (approximately 80 per cent) to the per cent transmission found in common optical materials like BK7 (approximately 92 per cent) over the visible region. We attribute the lower transmission in PR48 to a combination of surface scattering and increased absorption. At the present time, we do not know what fraction of the lower transmission is related to the surface quality resulting from sample polishing.

\subsection{Optical components}

Lenses made from optical plastics offer some advantages over lenses made from optical glass. For example, the manufacturing process is simpler, and the cost and weight are lower. However, plastics tend to absorb and scatter incident light to a greater level, and their scratch resistance is generally lower.

In the case of printed lenses, our results indicate that it is possible to print converging lenses with focal lengths that agree reasonably well with the thin lens equation. For example, it was determined that the focal length of the polished lens differs by 0.6 
Figure 9 A comparison between the measured (solid dots) and theoretical (red line) Fraunhofer diffraction pattern from (a) a printed grating with a $10 \mu \mathrm{m}$ periodicity, (b) a printed grating with a $25 \mu \mathrm{m}$ periodicity and (c) a printed grating with a $50 \mu \mathrm{m}$ periodicity. The data were taken with a red HeNe laser $(\lambda=632.8 \mathrm{~nm})$. Both the data and theory have been normalized to unity
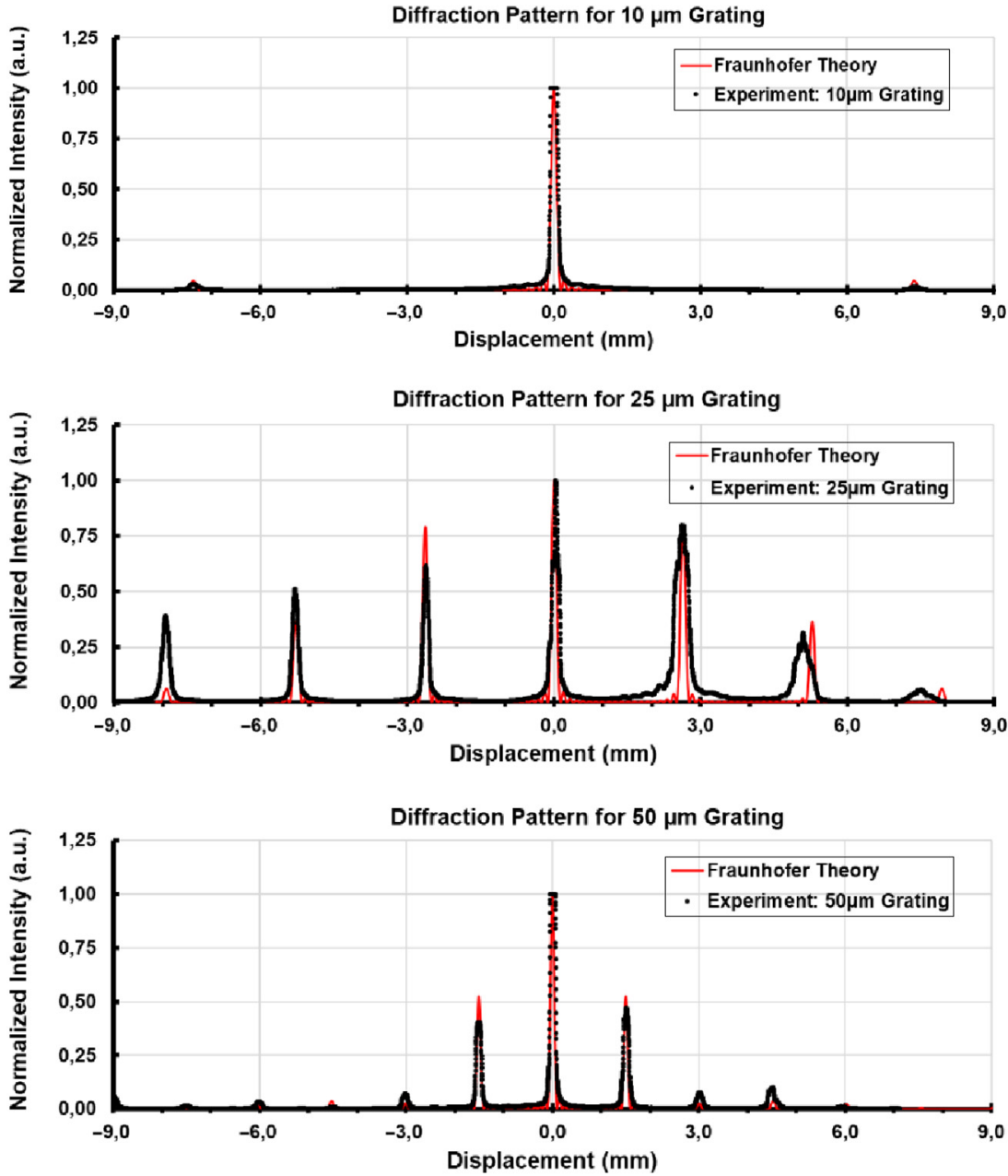

Table V Comparison of the layer thickness in the printed gratings to the effective values of $d$ and $w$ found by fitting the observed diffraction pattern to equation (9)

\begin{tabular}{lcc}
\hline $\begin{array}{l}\text { Printer setting } \\
\text { Layer thickness }[\mu \mathrm{m}]\end{array}$ & $\begin{array}{c}\text { From the diffraction pattern } \\
\text { Effective } d[\mu \mathrm{m}]\end{array}$ & \begin{tabular}{l} 
Effective $\boldsymbol{w}[\mu \mathrm{m}]$ \\
\hline 10
\end{tabular} \\
25 & 8.6 & 7.0 \\
50 & 24.0 & 6.3 \\
& 42.0 & 18.0 \\
\hline
\end{tabular}

per cent from the theoretical value of the lens. We believe the difference between the theoretical and experimental focal length is mainly due to the manual removal of material during the polishing process, which alters the radii of curvature of the lens.

There are inherent limitations to the $3 \mathrm{D}$ manufacturing process that affect the performance of lenses. Approximations to a curved surface in the design software, the printing resolution of the Autodesk Ember printer and the anisotropy due to printing in layers are issues (Monzón et al., 2017; Melgarejo et al., 2018). The performance of the lenses is also affected by internal imperfections in the printed material, in particular, the presence of bubbles and the inclusion of debris like dust or fibers suspended in air. In addition, the absorption of wavelengths in the blue/ultraviolet produces an undesirable yellowing in any printed part.

Surprisingly, diffraction gratings can be manufactured using DLP technology. The slits in a printed grating are formed at the interface between printed layers, presumably resulting from the additional cross-linking caused by the double exposure of each cured layer. Adhesion between two layers occurs when an already cured layer crosslinks with an uncured layer (Gong et al., 2015). It is thought that these adhesion regions present different optical properties compared to single cross-linked regions. This, in turn, produces an internal pattern that allows light to traverse certain regions of the material but not all of it. While the patterning of slits in a conventional grating is localized to the surface of a material, in a DLP-printed grating, an effective slit is formed throughout the thickness of the printed material. 
Figure $10 \mathrm{In}(\mathrm{a})$, an optical micrograph of a printed diffraction grating with a nominal layer thickness $10 \mu \mathrm{m}$. In (b), an optical micrograph of a printed diffraction grating with a nominal layer thickness of $25 \mu \mathrm{m}$. In (c), an optical micrograph of a printed diffraction grating with a nominal layer thickness of $50 \mu \mathrm{m}$. All micrographs taken at a magnification of $500 \times$. A scale bar in the bottom right of each image is equal to $30 \mu \mathrm{m}$

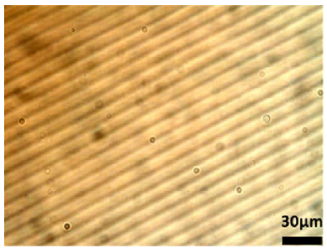

(a)

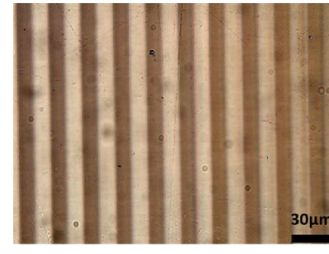

(b)

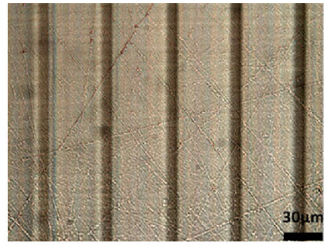

(c)

Table VI Compilation of the values for the average $<\mathrm{d}>$ and $<\mathrm{w}>$ from the micrographs taken with an optical microscope

\begin{tabular}{lcccc}
\hline Printer setting & \multicolumn{3}{c}{ Optical microscope images } \\
Layer thickness $[\mu \mathrm{m}]$ & $<\mathbf{d}>[\mu \mathrm{m}]$ & Uncertainty $( \pm)$ & $<\mathbf{w}>[\mu \mathrm{m}]$ & 6.9 \\
\hline 10 & 10.0 & 0.8 & 11.7 & 0.8 \\
25 & 25.4 & 0.8 & 8.8 & 0.8 \\
50 & 50.2 & 0.8 & 0.8 \\
\hline
\end{tabular}

Table VII Percentage difference between the effective values of $d$ and $w$ obtained by fitting the diffraction pattern to the values of $\langle d\rangle$ and $\langle w\rangle$ measured from optical micrographs

\begin{tabular}{lcc}
\hline $\begin{array}{l}\text { Printed layer } \\
\text { thickness }[\mu \mathrm{m}]\end{array}$ & $\begin{array}{c}\text { Percentage } \\
\text { difference in } \boldsymbol{d}[\%]\end{array}$ & $\begin{array}{c}\text { Percentage } \\
\text { difference in } \boldsymbol{w}[\%]\end{array}$ \\
\hline 10 & 15.1 & 1.4 \\
25 & 5.7 & 60.0 \\
50 & 17.8 & 68.6 \\
\hline
\end{tabular}

Regarding the diffraction gratings, the results in Tables V and VI show that the periodicity $d$ inferred from the diffracted intensity profiles and the periodicity $\langle d\rangle$ measured from optical micrographs agree well with the layer thickness setting of the Autodesk Ember printer. However, values for the effective slit width inferred from the diffraction data and the values measured from optical micrographs cannot in all cases yield reliable estimates for the effective width of the slits $w$.

\section{Conclusions}

Optical plastic resins combined with 3D printing technology provide many advantages over traditionally manufactured optical components, allowing parts with different shapes and internal patterns to be easily manufactured. This paper presented measurements of the refractive index and per cent transmission coefficient of cured PR48 resin. In addition, we have made optical elements such as prisms, lenses and diffraction gratings through DLP additive manufacturing. It is worth remarking that the manufacture of optical components using additive manufacturing must consider the characteristics of the material and the advantages and limitations these materials and processes present.

One of the most interesting results from this work was the manufacture of diffraction gratings using $3 \mathrm{D}$ printing. An analysis of the diffraction pattern produced by these printed gratings yielded estimates for the slit periodicity and effective slit width. These gratings are unique because the effective slit width fills the entire volume of the printed part. This aspect makes it possible to integrate two or more optical devices in a single printed part. For example, production of a lens combined with a diffraction grating. To the best of the authors' knowledge, no 3D-printed gratings using these techniques have been reported in academic literature and no reports of a fully characterized printed grating have been produced. This work further discusses the use of additive manufacturing techniques, particularly the DLP method, and studies the effect of layer thickness on the resulting gratings. The effects of layered polymerization on the final product are shown to affect the way in which light can be diffracted, hence producing a method for production of tunable and repeatable diffraction gratings.

\section{References}

Allnex (2013), "Allnex technical data sheet: Ebercyl 8210", available at: www.palmerholland.com/Assets/User/Documents/ Product/40741/4924/MITM00436.pdf

Aniwaa (2019), "Ember Autodesk", available at: www.aniwaa. com/product/3D-printers/autodesk-ember/ (accessed 7 January 2019).

Arkema (2014), available at ArkemaGPSSafetySummarySR494: available at: www.arkema.com/export/shared/.content/media/ downloads/socialresponsability/safety-summuries/PhotocureResins-SR-494-Alkoxylated-pentaerythritol-tetraacrylateGPS-2014-12-15-V0.pdf

Autodesk (2018), Ember Autodesk Standard Clear Prototyping Resin PR48, available at Autodesk: https://discourse-cdnsjc2. com/./1b755fa09bb75aae7395f2ffccd444c68b06f3ba.pdf 
Burns, M. (1993), Automated Fabrication: Improving Productivity in Manufacturing, Prentice Hall, Englewood Cliffs, NJ, ISBN:0131194623.

Chen, X., Liu, W., Dong, B., Lee, J., Ware, H., Zhang, H. and Sun, C. (2018), "High-speed 3D printing of millimeter-size customized aspheric imaging lenses with sub $7 \mathrm{~nm}$ surface roughness", Advanced Materials, Vol. 30 No. 18, p. 1705683.

Freeman, M.H. and Hull, C.C. (2003), Optics, ed., Butterworth Heinemann, Spain.

Furlan, W., Ferrando, V., Monsoriu, J., Zagrajek, P., Czerwińska, E. and Szustakowski, M. (2016), "3D printed diffractive terahertz lenses”, Optics Letters, Vol. 41 No. 8, pp. 1748-1751.

Gawedzinski, J., Pawlowski, M. and Tkaczyk, T. (2017), "Quantitative evaluation of performance of 3D printed lenses", Optical Engineering, Vol. 56 No. 8, p. 084110.

Gong, H., Beauchamp, M., Perry, S., Wooley, A. and Nordin, G. (2015), "Optical approach to resin formulation for 3D printed microfluidics", RSC Advances, Vol. 5 No. 129, pp. 106621-106632.

Hecht, E. (2017), Optics, 5th ed., Pearson Education.

Lee, J.H., Prud'Homme, R.K. and Aksay, I.A. (2001), "Cure depth in photopolymerization: experiments and theory", Fournal of Materials Research, Vol. 16 No. 12, pp. 3536-3544.

Li, Y., Linke, B.S., Voet, H., Falk, B., Schmitt, R. and Lam, M. (2017), "Cost, sustainability and surface roughness quality - a comprehensive analysis of products made with personal 3D printers", CIRP Fournal of Manufacturing Science and Technology, Vol. 16, pp. 1-11.

Melgarejo, L.D.V., García, J., Reifenberger, R.G. and Newell, B. (2018), "Manufacture of lenses and diffraction gratings using DLP as an additive manufacturing technology", ASME 2018 Conference on Smart Materials, Adaptive Structures and Intelligent Systems, American Society of Mechanical Engineers, pp. V002T08A004-V002T08A004.

Monzón, M., Ortega, Z., Paz, R. and Ortega, F. (2017), "Anisotropy of photopolymer parts made by digital light processing”, Materials, Vol. 10 No. 1, p. 64, doi: 10.3390/ ma10010064.

Pedrotti, F.L., Pedrotti, L.M. and Pedrotti, L.S. (2017), Introduction to Optics, Cambridge University Press.

Riahi, M. (2016), "Fabrication of corner cube array retroreflective structure with DLP-based 3D printing technology", Optical Review, Vol. 23 No. 3, pp. 442-447, doi: 10.1007/s10043-016-0212-z.

Skliutas, E., Kasetaite, S., Jonušauskas, L., Ostrauskaite, J. and Malinauskas, M. (2018), "Photosensitive naturally derived resins toward optical 3D printing", Optical Engineering, Vol. 57 No. 4, p. 041412.

Squires, A.D., Constable, E. and Lewis, R.A. (2015), “3D printed terahertz diffraction gratings and lenses", fournal of Infrared, Millimeter, and Terahertz Waves, Vol. 36 No. 1, pp. $72-80$.

Sung, J.L., Jeang, J., Lee, C.H. and Shih, W.C. (2015), "Fabricating optical lenses by inkjet printing and heat assisted in situ curing of polydimethylsiloxane for smartphone microscopy", Fournal of Biomedical Optics, Vol. 20 No. 4, p. 47005.

\section{Corresponding author}

José M. Garcia-Bravo can be contacted at: jmgarcia@, purdue.edu 